\title{
A Novel and Atypical Nf-Kb Proinflammatory Program Regulated by A Camkii-Proteasome Axis Is Involved in the Early Activation of Muller Glia by High Glucose
}

\section{Diego Ph.D. Diego Sbardella ( $\nabla$ diego.sbardella@fondazionebietti.it )}

IRCSS Fondazione G B Bietti per lo Studio e la Ricerca in Oftalmologia ONLUS https://orcid.org/00000002-9924-7761

\section{Grazia Raffaella Tundo}

IRCSS Fondazione G B Bietti per lo Studio e la Ricerca in Oftalmologia ONLUS

\section{Alice Mecchia}

Fondazione Bietti: IRCSS Fondazione G B Bietti per lo Studio e la Ricerca in Oftalmologia ONLUS

\section{Camilla Palumbo}

University of Rome Tor Vergata: Universita degli Studi di Roma Tor Vergata

\section{Maria Grazia Atzori}

University of Rome Tor Vergata: Universita degli Studi di Roma Tor Vergata

\section{Lauretta Levati}

Istituto Dermopatico dell'Immacolata IRCCS: Istituto Dermopatico dell'Immacolata Istituto di Ricovero e Cura a Carattere Scientifico

\section{Anna Maria Caccuri}

University of Rome Tor Vergata: Universita degli Studi di Roma Tor Vergata

\section{Paolo Cascio}

\section{Politecnico di Torino}

\section{Pedro Lacal}

Istituto Dermopatico dell'Immacolata Istituto di Ricovero e Cura a Carattere Scientifico

\section{Grazia Graziani}

University of Rome Tor Vergata: Universita degli Studi di Roma Tor Vergata

\section{Monica Varano}

Fondazione Bietti: IRCSS Fondazione G B Bietti per lo Studio e la Ricerca in Oftalmologia ONLUS

\section{Massimiliano Coletta}

University of Rome Tor Vergata: Universita degli Studi di Roma Tor Vergata

\section{Maria Cristina Parravano}

Fondazione Bietti: IRCSS Fondazione G B Bietti per lo Studio e la Ricerca in Oftalmologia ONLUS 
Research

Keywords: Diabetic retinopathy, Muller glia, NF-kB pathway, Proteasome, CamKII

Posted Date: December 9th, 2021

DOI: https://doi.org/10.21203/rs.3.rs-1116573/v1

License: (c) (i) This work is licensed under a Creative Commons Attribution 4.0 International License. Read Full License 


\section{Abstract}

\section{Background}

Diabetic retinopathy (DR) is a microvascular complication of diabetes with a heavy impact on the lifequality of subjects and with a dramatic burden for health and economic systems on a global scale.

Although the pathogenesis of DR is largely unknown, several preclinical data have pointed out to a main role of Muller glia, a cell type which spans across the retina layers providing nourishment and support for Retina Ganglion Cells (RGCs), in sensing glycemia and in acquiring a proinflammatory polarization in response to this insult.

\section{Results}

By using a validated experimental model of DR in vitro, the rMC1 cells challenged with high glucose, we uncovered the induction of an early (within minutes) and atypical NF-kB signalling pathway regulated by a CamKII-proteasome axis. Phosphorylation of proteasome subunit Rpt6 (at serine 120) by CamKII stimulated the accelerated turnover of $\mathrm{IkBa}$ (i.e., the natural inhibitor of p65-50 transcription factor), regardless of the phosphorylation at serine 32 which labels canonical NF-kB signalling. This event allowed the p65-p50 heterodimer to migrate into the nucleus and to induce the selective transcription of IL-8, II-1 $\beta$ and MCP-1. Pharmacological inhibition of CamKII or proteasome stopped this proinflammatory program, whereas introduction of a Rpt6 phospho-dead mutant (Rpt6-S120A) stimulated a paradoxical effect on NF-kB probably through the activation of a compensatory mechanism which may involve phosphorylation of $20 \mathrm{~S}$ a4 subunit.

\section{Conclusions}

This study introduces a novel pathway of MG activation by high glucose and casts some light on the biological relevance of proteasome post-translational modifications in modulating pathways regulated through targeted proteolysis.

\section{Bullet Points}

- High glucose quickly induces an atypical NF-kB proinflammatory program.

- CamKII phosphorylation of Rpt6 subunit of the proteasome stimulates IkBa turnover and p65-p50 release.

- Pharmacological inhibition of either Camkll or proteasome blocks this pathway

\section{Introduction}

Diabetic retinopathy (DR) is a microvascular complication of diabetes which often leads to irreversible blindness, a clinical outcome which carries a heavy burden in terms of quality of life of affected subjects 
and economic-social costs on a global scale ${ }^{1,2}$.

DR pathogenesis evolves through two stages, referred to as non-proliferative (NPDR) and proliferative DR (PDR). NPDR is characterized by increased vascular permeability followed by haemodynamic alterations of choroidal and retinal vasculature, whereas the hallmark of PDR is neo-angiogenesis ${ }^{1}$.

Muller glia (MG), a cell type which span across the neuroretina providing mechanical and nutritional support to the highly specialized retina neurons, has long been matter of studies aimed at figuring out DR pathogenesis, as it was reported to acquire a reactive phenotype in response to hyperglycaemia in rat models of DR and in human retina isolated post-mortem ${ }^{3-7}$.

Furthermore, prolonged (days) cultivation of $M G$ cells in high glucose ( $\geq 25 \mathrm{mmol} / \mathrm{L}$ ) medium was reported to induce the synthesis and secretion of: a) pro-inflammatory cytokines (interleukin $1 \beta$ [IL-1 $\beta$ ], tumour necrosis factor a [TNFa], interleukin-8 [IL-8], monocyte chemoattractant protein-1 [MCP-1] and vascular endothelial growth factor (VEGF), a master regulator of neo-angiogenesis ${ }^{8-10}$.

Nuclear factor-kB (NF-kB) is a master inflammatory pathway which regulates the transcription of these genes. In the canonical pathway, [for a comprehensive description of pathway refer to ${ }^{11-16}$ ], the NF-kB core transcription factor, composed by a p65 and p50 heterodimer, is kept inactive in the cell cytosol through the binding with the inhibitor of kinase $\mathrm{B}-\mathrm{a}(\mathrm{I} \mathrm{kBa})$, a family of natural inhibitors.

$\mathrm{IkBa}$ is a natively unfolded protein which short half-life (few minutes) due to the presence of a PEST sequence in the $\mathrm{C}$-terminus which promotes its ubiquitin-independent degradation by the proteasome ${ }^{11}$. When complexed with p65-p50, the PEST sequence is masked and lkBa gains stability and longer half-life (up to $8 \mathrm{~h}$ ) ${ }^{11,14}$. Release of p65-p50 then occurs through a signalling cascade induced by proinflammatory stimuli (e.g., TNFa and IL-1 $\beta$ or LPS) which involves the inhibitor of kappa B kinase (IKK).

Once activated, IKK phosphorylates p65-p50-bound IkBa at serine residues 32 and 36 promoting its ubiquitylation by the Tprc $\beta$ E3ligase and proteasome-mediated degradation. This renders the p65-p50 heterodimer free to enter the nucleus, where it binds to the promoter sequences of pro-inflammatory and antiapoptotic genes, including $\mathrm{lkBa}$, according to a negative feedback mechanism that has evolved to shut the transcriptional activity of this pathway off 11,12 .

Hence, regulation of NF-kB further requires a functional Ubiquitin Proteasome System (UPS), a major intracellular proteolytic pathway that stands at the crossroad of cell metabolism ${ }^{17-20}$.

In the canonical structural configuration,, the proteasome is made up by the $20 \mathrm{~S}$, a hollow barrel-shaped particle composed of four heptameric rings, two outer a rings and two inner $\beta$, which house the catalytic subunits (i.e., chymotrypsin-like, trypsin-like and caspase-like) complexed, at one or both a-ends, with the $19 \mathrm{~S}^{18,21,22}$. The $19 \mathrm{~S}$ is arranged into the base (Rpt(s) subunits) and the lid (Rpn(s) subunits) 
subassemblies which couple the affinity-recognition and the ATP-hydrolysis, to unfold and pull the polyubiquitylated proteins down into the 20 s catalytic chamber ${ }^{22-24}$.

Proteasome composition is constantly tuned to meet the metabolic need of the cells through binding with Regulatory Particles (RPs) others than the 19S which modulate its functional properties, as well as through post-synthetic modifications of subunits ${ }^{25-2728,29}$. This last possibility is an emerging topic in proteasome biology, as phosphorylation, acetylation, glycosylation of selected subunits are being unveiled in pathophysiological conditions, though the functional significance is often unclear 30,31 .

Herein, we have carried out a characterization of the early dynamics of canonical NF-kB signaling and proteasome modulation by a hyperglycemic insult in $\mathrm{rMC1}$ cells, a validated experimental model of Muller glia. High glucose $(25 \mathrm{mmol} / \mathrm{L})$ delivery was found to quickly induce an atypical nuclear translocation and transcriptional activation of p65-p50 through a serine 32 phosphorylation-independent clearance of IkBa by the proteasome. This pathway seems activated through phosphorylation of the 19S Rpt6 subunit at serine 120 by a calcium-dependent calmodulin kinase (CamKII), an event that triggers the bulk degradation of intracellular poly-ubiquitylated substrates, likely shifting, through targeted proteolysis, the equilibrium of biological reactions regulated by short-lived proteins.

\section{Results}

\section{Transcription of proinflammatory cytokines is induced early by high glucose concentrations in rMC1 cells}

We preliminarily noticed that immunostaining of the $17 \mathrm{kDa}$ IL-1 $\beta$ fragment (i.e., the biologically active species) was very robust in whole lysates of $\mathrm{rMC} 1$ challenged with $25 \mathrm{mmol} / \mathrm{L}$ glucose (referred to as high glucose throughout the text) for up to $2 \mathrm{~h}$ (Fig. 1A). The intensity of cytokine immunostaining was significantly lower in lysates of cells stimulated with $5 \mathrm{mmol} / \mathrm{L}$ glucose or mannitol, used as hyperosmolar control (referred to as "controls"). Conversely, immunostaining of IL-12 (Fig. 1A) and IL-10 (undetectable, data not shown), which are not regulated by NF-kB, was unaltered by treatments over the same time-interval.

To address whether the IL-1 $\beta$ increase reflected the transcriptional upregulation and not a post-synthetic mechanism (e.g., increased processing of pre-IL-1 $\beta$ or impaired secretion), a RT-PCR analysis of rMC1 challenged with $25 \mathrm{mmol} / \mathrm{L}$ glucose and controls for $40 \mathrm{~min}$ was undertaken. Compared to control cells, cells challenged with high glucose displayed a $\sim 2$-fold increase of IL-1 $\beta$ transcript (Fig. 1B). Thus, additional cytokines were enrolled in the study: IL-8 ( 5-fold increase) and MCP-1 ( 3-fold increase) turned out to be induced to the greatest extent in high glucose challenged cells. Conversely, transcription of IL-6 and TNFa was not induced (Fig. 1B).

\section{An atypical NF-kB pathway is activated within minutes after rMC1 challenge with high glucose}


To investigate whether high glucose may actually trigger an early activation of NF-kB, a time-course analysis of $\mathrm{lkBa}$ phosphorylation at serine $32[\mathrm{plkBa}(\mathrm{ser} 32)]$ in whole cell lysates of $\mathrm{rMC} 1$ challenged for 10,20 and 40 min with $25 \mathrm{mmol} / \mathrm{L}$ glucose and low glucose or mannitol was performed by $\mathrm{Wb}^{11-13}$. Compared to control cells, plkBa(ser32) immunostaining dropped after $10 \mathrm{~min}$ of stimulation with highglucose, and was persistently decreased within the time-interval analysed (Fig. 2A). Immunostaining of unphosphorylated IkBa dropped as well, but, with respect to plkBa(ser32), it showed a 10 min delay: in fact, compared to control cells, the greatest decrease of total IkBa was documented 20 min after high glucose delivery (Fig. 2A and Suppl. Fig. 1). Thus, the plkBa(ser32)/lkB ratio, usually very high during canonical NF-kB signalling, was markedly decreased at $10 \mathrm{~min}$ of high glucose challenge, but unaltered at 20 and 40 min (Fig. 2A).

To rule out the existence of unprecedented lkBa phospho-sites, a phos-Tag analysis was set up. Separation of lysates from control and high glucose treated cells allowed to detect a band pair compatible with unphosphorylated and phosphorylated IkBa (see lower and upper band in overexposed blot, Fig. 2B). However, high glucose did not cause any obvious increase of delayed-mobility species at any time-point investigated (Fig. 2B).

To verify whether plkBa(ser32) and IkBa drop was followed by nuclear translocation of p65-p50, the nuclear and cytosolic fractions of $\mathrm{rMC} 1$ stimulated with $25 \mathrm{mmol} / \mathrm{L}$ glucose and controls for 30 min were isolated and analysed by $\mathrm{Wb}$. Immunostaining of p65 and p50 highlighted a significant increase of the two proteins in the nuclear fraction of high glucose $v s$ control cells, mirrored by a drop in the corresponding cytosolic fraction (Fig. 2C). Lamin-D2 and $\beta$-tubulin immunostaining confirmed the identity of the two fractions and a negligible reciprocal contamination (Fig. 2C).

To further validate NF-kB activation the DNA binding activity of p65 was assayed in the nuclear extracts of cells challenged with high glucose and controls for $30 \mathrm{~min}$ by using a commercially available ELISAimmunoassay (Fig. 2D). The NF-kB DNA binding ability, measured by the absorbance at $470 \mathrm{~nm}$ (O.D.), was about 2-fold higher in high glucose than in control cells nuclear extracts (Fig. 2D).

\section{A second cycle of canonical NF-kB activation is observed at later time points in rMC1 cells exposed to high glucose}

Whole cell lysates were collected from $\mathrm{rMC} 1$ challenged with high glucose and low glucose or mannitol for 1, 2 and $3 \mathrm{~h}$ to figure out whether cytokines, once released, induced a canonical NF-kB signalling following a proinflammatory pathway. Compared to control cells, immunodetection of plkBa(ser32) progressively increased 2-3h after high glucose delivery, whilst, IkBa dropped significantly (Fig. 3). Hence, this time, the plkBa(ser32)/lkBa ratio was greater in high glucose than in control cells.

To confirm the occurrence of a canonical NF-kB signalling, filters were probed for unphosphorylated and phosphorylated IKK $\beta$ [at serine 180, referred to as pIKK $\beta(\operatorname{ser} 180)$ ], and unphosphorylated and phosphorylated p65 (at serine 536, referred to as p65(ser536] (Fig. 3). After 2-3h of stimulation, pIKK $\beta$ (ser180) turned out to be detectable, in the absence of any variation of total IKK $\beta$. Similarly, 
phosphorylation of p65(ser536) significantly increased after 2-3h of high glucose stimulation with respect to control cells. Also in this case, no modulation of the unphosphorylated p65was observed (Fig. 3). Thus, the p65(ser536)/p65 ratio was increased in high glucose treated (in particular at 2h) than in control cells.

\section{Proteasome bulk activity is stimulated by $25 \mathrm{mmol} / \mathrm{L}$ glucose in $\mathrm{rMC} 1$ cells.}

Since $\mathrm{lkBa}$ is a prototypical proteasome substrate, to shed light on the NF-kB early activation,we verified whether high glucose delivery for 10-40 min actually induced a drop of ubiquitylated proteins by $\mathrm{Wb}$. Compared to control cells, high glucose induced a robust decrease of ubiquitinylated proteins and a slight drop of p21 and p53, two reporter substrates of proteasome, over this time interval (Fig. 4A). To rule out alterations of the ubiquitylation pathway, cells were pre-treated with $500 \mathrm{nM}$ epoxomicin (or DMSO vehicle) for $1 \mathrm{~h}$ before administering the high glucose challenge for $40 \mathrm{~min}$. The proteasome inhibitor increased the basal content of ubiquitylated proteins in control cells and prevented their drop in high glucose treated cells (Fig. 4B).

To address whether bulk proteasome activity was induced by glucose uptake, crude cell extracts of rMC1 challenged with high glucose and low glucose or mannitol for 10, 20 and 40 min were assayed for the kinetics of Suc-LLVY-amc hydrolysis, a synthetic peptide of chymotrypsin-like activity (Fig. 4C). The cleavage rate of LLVY-amc was faster by extracts of cells challenged with high glucose at each timepoint analysed (Fig. 4C). To further validate this finding, proteasome particles were separated by nativegel electrophoresis and probed with $75 \mu \mathrm{M}$ LLVY-amc (Fig. 4D). Compared to control cells, high glucosestimulated $\mathrm{rMC1}$ displayed a robust hyper-activation of the capped assemblies (i.e., 30S-26S) which reached a peak around 20 min after stimulus delivery (Fig. 4D).

Immunostaining with an anti-Rpt5 (i.e., a 19S subunit) antibody clarified that the capped particles content was actually increased in high glucose stimulated cells with respect to control cells, in association with a drop of free 19S (Fig. 4D, bottom panel). To rule out uneven gel loading, crude cell extracts were further run by canonical $\mathrm{Wb}$ and probed with antibodies raised against a4 (i.e., 20S), Rpt5 and Rpt6 (i.e., 19S) (Fig. 4D, bottom panel).

Thereafter, proteasome proteolytic activity in living cells was further probed with TED, a quenched fluorogenic peptide of proteasome, according to a method validated elsewhere ${ }^{32}$.

To meet all the chronological criteria (TED properties and timing of proteasome activation by glucose), rMC1 were first pre-treated with $500 \mathrm{nM}$ epoxomicin for $1 \mathrm{~h}$. Thereafter, high glucose and controls were administered and, after additional 5 min of incubation, $17 \mu \mathrm{M}$ TED peptide was added to the culture medium and fluorescence release recorded for a total of $20 \mathrm{~min}$ after high glucose delivery. Following TED administration, fluorescence intensity quickly increased under all experimental conditions tested. However, after $15 \mathrm{~min}$ from TED addition (that is $20 \mathrm{~min}$ from glucose administration), whilst TED fluorescence reached a plateau in low-glucose or mannitol-treated cells, it displayed an additional steep increase in high glucose-stimulated cells (Fig. 4E). 
High glucose induces the phosphorylation of the Rpt6 subunit of the 19S.

The rapidity through which proteasome activation took place was suggestive of a post-synthetic modification, such as phosphorylation, on some proteasome subunits.

To test this possibility, a phos-Tag assay was set up to screen the electrophoretic mobility of a panel of $19 S$ subunits for which phospho-sites have been described, such as Rpn6, Rpt3, Rpt5 and Rpt6 ${ }^{33-37}$, in rMC1 stimulated with $25 \mathrm{mmol} / \mathrm{L}$ glucose and controls for 20 and $40 \mathrm{~min}$. Rpn 6 and Rpt5 immunostaining did not reveal any delayed mobility of species others than the unphosphorylated one (Fig. 5). Rpt6 immunostaining highlighted a delayed mobility of two bands (black arrow), which, indeed, were nicely detectable under all the experimental conditions. However, these phosphorylated species stained much stronger in high glucose-treated than in controls-treated cells, and this was paralleled by a drop of the intensity of the unphosphorylated species. Conversely, Rpt3 showed delayed-mobility species but no altered ratio between phospho- and unphosphorylated bands under any of the experimental conditions tested (Fig. 5).

Whole cell lysates were then probed with a phospho-specific antibody raised against Rpt6 phosphorylated at serine 120, a major phospho-site. Immunodetection of phospho- and unphospho-Rpt6 confirmed the presence of a significant basal level of phosphorylation of this subunit under all experimental conditions, but, compared to untreated $\mathrm{rMC1}$, further highlighted a robust increase of the pRpt6(Ser120)/Rpt6) ratio in cell lysates harvested after 20 and 40 min of high glucose stimulation (Fig. 5).

\section{CamKII but not PKA phosphorylates Rpt6 and stimulates bulk proteasome activity in MG}

To identify the kinase that actually phosphorylated Rpt6, we first focused on the calcium-dependent calmodulin Kinase II (CamKII) and protein kinase A (PKA) by using two specific inhibitors called Inhibitor XII (Inh. XII) and KT5720, respectively. First, we ruled out the presence of major effects of the inhibitors on proteasome activity under basal growth condition (Suppl. Fig. 2A). Then, after a $2 \mathrm{~h}$ pre-treatment with the two inhibitors (each delivered at $10 \mu \mathrm{M}$ ) (or DMSO vehicle), rMC1 cells were stimulated with $25 \mathrm{mmol} / \mathrm{L}$ glucose or controls for 20 min and crude cell extracts probed for the kinetics of LLVY-amc hydrolysis (Fig. 6A). Cells treated with DMSO or KT5720 displayed an increase of LLVY-amc hydrolysis by high glucose comparable to that reported previously (see Fig. 4B) (Fig. 6A). Conversely, Inh. XII robustly opposed proteasome activation at 20 min of the high glucose challenge (Fig. 6A).

This finding was confirmed also by native-gel electrophoresis (Fig. 6B). Again, whilst DMSO and KT5720 did not alter the proteolytic activation of the capped assemblies by $25 \mathrm{mmol} / \mathrm{L}$ glucose (see Fig. 4D), Inh. XII prevented particle activation by sugar intake, both at 20 and 40 min after challenge induction (Fig. 6B). Interestingly, unlike DMSO and KT5720, Inh. XII further reduced the engagement of the free 19S in assembling the capped particles in the presence of $25 \mathrm{mmol} / \mathrm{L}$ glucose (Fig. 6B). 
The phos-Tag approach then confirmed that Inh. XII blocked the phosphorylation of Rpt6, whilst DMSO and KT5720 did not, as the delayed-mobility band staining positive for Rpt6 was increased only in the combined presence of the two latter compounds and of high glucose (Fig. 6C).

Moreover, in cells challenged with high glucose, the immunodetection of pRpt6(ser120), as well as the pRpt6(ser120)/Rpt6 ratio were markedly more robust in the presence of DMSO and KT5720 treated-cells than in Inh. XII-treated cells which further displayed a drop of basal phosphorylated Rpt6 (Fig. 6D).

\section{Inhibition of CamKII and proteasome blocks NF-kB activation by high glucose in rMC1 cells}

We then tried to address whether proteasome and NF-kB activation were actually part of the same pathway.

First, we investigated the effects of Inh. XII, KT5720 and epoxomicin on the canonical NF-kB signalling and on CamKII content over $2 \mathrm{~h}$ of stimulation under basal growth conditions (Suppl. Fig. 2B). Whole lysates were analysed by Wb revealing no alteration of IkBa and IKK $\beta$ and CamKII (the presence of the $a$ and $\beta$ isoforms was unveiled) content (Suppl. Fig. 4). Immunostaining of plkBa(ser32), was unaltered by Inh. XII and KT5720 but displayed a robust increase at $2 \mathrm{~h}$ of epoxomicin stimulation.

Then, the turnover of IkBa after 20 min of high glucose challenge was investigated after having pretreated $\mathrm{rMC} 1$ with each inhibitor. BAPTA-AM $(10 \mu \mathrm{M})$, a validated cell-permeable $\mathrm{Ca}^{2+}$ chelator was also tested in this study. With respect to control cells, induction of IkBa decrease by high glucose was documented only in the presence of DMSO and of KT5720. Addition of either Inh. XII, epoxomicin or BAPTA-AM blocked the degradation of IkBa (Fig. 7A). To figure out whether inhibition of CamKII by Inh. $\mathrm{XII}$ and of proteasome by epoxomicin was effective also in inhibiting the transcriptional upregulation of proinflammatory cytokines, IL-8, IL-1 $\beta$ and MCP-1 transcripts were assayed by RT-PCR in IMC1 cells exposed or not to $25 \mathrm{mmol} / \mathrm{L}$ glucose for $40 \mathrm{~min}$ (Fig. 7B). Pre-treatment with Inh. XII or epoxomicin almost abolished the upregulation of the cytokines in by high glucose (Fig. 7B) while DMSO had no effect (see comparison with Fig. 1B).

To test whether CamKII, but not PKA inhibition was effective also in halting plkBa phosphorylation at serine 32 during the second cycle of NF-kB activation (see Fig. 2), rMC1 cells, pre-treated with Inh. XII and KT5720 were challenged with high glucose for 2 and 3h (Fig. 8). Compared to control cells, plkB(ser32) immunostaining in the presence of high glucose was increased in cells treated with DMSO or KT5720, whereas Inh. XII drastically opposed the accumulation of this phosphorylated protein (Fig. 8).

Influence of Rpt6 mutagenesis on early NF-kB activation in MG exposed to high glucose.

To clarify the role of Rpt6 phosphorylation in driving the early NF-kB activation, rMC1 were stably transfected with a plasmid encoding a phospho-dead mutant Rpt6 in which serine 120 was replaced by alanine (hereafter referred to as Rpt6_S120A) and with the empty vector (hereafter referred to as Vector) as internal control. Two independent clones for Vector and Rpt6_S120A were generated. 
Following transfection, the Rpt6 immunostaining was greater in Rpt6_S120A than Vector cells, whilst that of additional Rpt(s) subunits was not, and pRpt(ser120) was significantly reduced in mutated clones (Suppl. Fig. 3A).

Surprisingly, Rpt6_S120A clones displayed a 2-fold increase of chymotrypsin-like activity on LLVY-amc with respect to Vector clones (Suppl. Fig. 3B). Furthermore, the capped assemblies and, most notably, the free 20S of Rpt6_S120A clones were significantly more active than those isolated from Vector cells, as from native-gel studies (Suppl. Fig. 3C).

Probing of filters with an anti-Rpt3 antibody made it clear that the capped particles immunostaining was markedly increased, especially that of the $30 \mathrm{~S}$, whereas the free 19S content was significantly reduced in Rpt6_S120A vs Vector clones. Conversely, the 20S immunostaining by an anti-a4 antibody was unaltered between clones, implying that the free 20S of Rpt6_S120A clones was hyperactive (Suppl. Fig. 3D).

Since the mass/charge electrophoretic pattern of the free 20S argued against the binding of an alternative regulatory particle $\mathrm{e}^{38,39}$,to interpret the $20 \mathrm{~S}$ hyperactivation whole cell lysates were of all clones were run in parallel by phos-Tag and Wb. Filters were probed with an anti-a4 and an anti-pan-a20S antibody, that targets 6 out of 7 a-subunits except for a4, producing a typical smear. The phos-Tag approach highlighted (Suppl. Fig. 3E) that a delayed-mobility species of a4 was missing out in Rpt6_S120A clones in the absence of variation of total a4 (Suppl. Fig. 3E). By probing the same filters with the pan-a20S antibody it emerged that the pattern of the six subunits was unaltered, although in the former case separation of individual subunits was unsuccessful probably due to the presence of constitutive phosphorylation of some subunits which altered the migration pattern superimposing at least of them (Suppl. Fig. 3E).

To address the consequences of proteasome activation on the dynamics of NF-kB, a panel of proteins involved in the pathway were assayed by Wb. Surprisingly, IkBa displayed a robust drop in Rpt6_S120A clones (Suppl. Fig. 4A). Conversely, plkBa(ser32) was significantly increased and p65 unaltered (Suppl. Fig. 4A). Yet, the poly-ubiquitinylated proteins were slightly and not significantly increased in the Rpt6_S120A clones together with p21, whilst p53 was significantly increased in these cells (Suppl. Fig. 4B).

Based on the lkBa results, the transcription of proinflammatory cytokines was then assayed by RT-PCR. Surprisingly, the IL-8 mRNA was found to be nearly 5-fold higher in Rpt6_S120A vs Vector clones, whereas MCP-1 did show a nearly 3.5-fold increase (Suppl. Fig. 4C). IL-1 $\beta$ (data not shown), instead, was characterized by a significant inter-experimental variability which did not allow to draw unambiguous conclusions.

\section{Discussion}

Herein, we describe an early and atypical pathway of p65-p50 transcriptional activation by high glucose in rMC1 whose distinctive feature is the lack of an obvious IkBa phosphorylation, seemingly replaced by 
the CamKII-dependent phosphorylation of the 19S Rpt6 subunit. Furthermore, we have confirmed the involvement of NF-kB canonical pathway in $\mathrm{rMC} 1$ proinflammatory polarization, but we provide evidence that, at least under the experimental conditions tested, this pathway is triggered much earlier than originally thought.

Muller glia cells undoubtedly play a significant role in the pathological retinal remodelling of diabetic subjects, as this cell type has been continuously reported to acquire morpho-functional markers of activation in the presence of high glucose concentrations and, notably, to express and secrete a plethora of pro-inflammatory cytokines and neurotransmitters $5,7,40,41$. Remarkably, by virtue of their anatomical and functional proximity, MG, pericytes and endothelial cells, are supposed to constitute a neurovascular unit inside which the metabolic alterations are readily transduced across the cell lineages 42 .

NF-kB is a highly conserved proinflammatory pathway which serves crucial biological activities for neuron homeostasis ${ }^{11,14,15}$. In the canonical pathway, p65-p50 transcriptional activation is dependent on a thermodynamic equilibrium between the availability of free $\mathrm{lkBa}$, bound $\mathrm{lkBa}$, the rate of its phosphorylation by proinflammatory signalling cascades and kinetics of proteasome clearance ${ }^{11}$. Biochemical and mathematical studies have provided elegant evidence of how the artificial increase of free IkBa half-life, through mutagenesis of the PEST sequence, turns out into a reduced rate of p65-p50 activation ${ }^{11}$.

In this study, NF-kB activation appears to be strictly dependent on CamKII and proteasome, since inhibition of either one of these two enzymatic activities led to a blockade of the pathway.

Whilst the occurrence of CamKII activation by $\mathrm{Ca}^{2+}$ quickly after high glucose delivery is supported by previous studies in $\mathrm{rMC} 1$ which highlighted the involvement of $\mathrm{P} 2 \mathrm{X} 7$ purinergic receptors in the dynamics of $\mathrm{Ca}^{2+}$ flux, the contribution of proteasome appears to be currently drawn only through thermodynamic assumptions 47 .

Bulk proteasome activity is supposed to be very low in comparison with the effective concentration of assembled particles, as they mostly lie in a substrate-engagement competent but proteolytic-incompetent state in living neurons ${ }^{48}$. The rate-limiting step of substrates degradation through the UPS has been long considered to be the Ub conjugation by E3-ligases, but Ub-tagged substrates are thought to bind constantly to catalytic-incompetent proteasome assemblies without being digested ${ }^{30}$. In this framework, proteasome phosphorylation is a post-synthetic mechanism which quickly increases the pool of catalyticcompetent particles and the velocity of poly-ubiquitylated short-lived substrates processing, orienting the kinetics of metabolic pathways regulated through targeted proteolysis $19,31,33,35,37,49$.

In this regard, NF-kB and the kinetics of $\mathrm{kBBa}$ binding and dissociation with p65-p50 represent an ideal thermodynamic equilibrium by which the enzyme (e.g., proteasome) hyper-activation subtracts the bioavailability of a reactant, in this case (we hypothesize) the constitutively phosphorylated IkBa, which is readily ubiquitylated and digested, shifting the whole equilibrium toward IkBa consumption and p65-p50 
release. We must suppose that proteasome hyperactivation pushes forward the lkBa-plkBa equilibrium, serving as a kinetic activator of a pathway regulated by the dissociation rate constant of $\mathrm{lkBa}$, which indeed occurs in few minutes ${ }^{50}$. Although it may be determined by additional factors, the 10 min delay between plkBa(ser32) and lkBa decrease after high glucose delivery may represent a further clue for this thermodynamic-driven equilibrium. ${ }^{51,52}$. However, it is also possible that the $26 \mathrm{~S}$ proteasome degrades free $\mathrm{lkBa}$ in the absence of ubiquitylation, but the equilibrium would proceed as well toward the direction proposed. Nevertheless, a possible involvement of phosphatasesin decreasing the plkBa(ser32) content is unlikely, as this occurrence would reasonably promote $\mathrm{kBBa}$ stabilization and retention of p65-p50 into the cytosolic compartment.

Although speculative, the synthesis and secretion of proinflammatory cytokines during the first round of transcriptional activation would act as an autocrine and paracrine stimulus for cyclic NF-kB activation which follows a canonical proinflammatory pathway. Nevertheless, the inhibition of the second round of NF-kB activation obtained through inhibition of CamKII, may indirectly support this possibility, although, at this stage, it cannot be ruled out that additional pathways, regulated by CamKII, might be responsible for this phenomenon. It is further worth pointing out that the pattern of cytokines transcribed is suggestive of a tailored regulation of p65-p50 activity, probably through post-synthetic modifications, as IL- 6 and TNF-a, which are commonly induced together with IL- $1 \beta$, in this case were not ${ }^{34,53}$.

The introduction of the Rpt6_S120A phospho-dead mutation is likely followed by a metabolic adaptation of the cells which involves both the capped assemblies, and most notably, the free 20S, which was a very unexpected finding also because the same mutation was reported to decrease basal proteasome activity in other eukaryotic systems ${ }^{34,53}$. Nonetheless, it is worth pointing out that Rpt6 looks to be phosphorylated at a significant basal level in rMC1, suggesting that this post-synthetic modification is of great relevance for the metabolism, at least for this cell type and that its absence induces paradoxical effects through compensatory mechanisms. One of them may involve the a4phosphorylation, which was previously reported inhibit $20 \mathrm{~S}$ activation ${ }^{54}$. In this respect, while this manuscript was being written, transgenic mice harbouring the Rpt6_S120A mutation displayed minimal defects on synaptic plasticity and learning, in spite of previous evidences ex vivo that these brain processes were regulated through this PTM, suggesting that this mutation might activate still unknown compensatory mechanisms ${ }^{55}$.

Thus, we very preliminary hypothesize that phosphorylation of Rpt6 could be the specific posttranslational modification coupling high glucose stimulation and proteasome activation of $\mathrm{rMC}$. However, this could represent one out of different proteasome PTMs serving roles for NF-kB regulation and it would be of broader biological relevance to figure out whether modulation of proteasome activity, regardless of the mechanism (e.g., PTMs, drugs, etc.) through which it is reached out, foster alternative signalling networks of pathways regulated by targeted proteolysis, such as NF-kB; this possibility was somewhat theorized by the "load vs capacity" model ${ }^{56}$.

In conclusion, if this molecular scenario here depicted will be confirmed by additional experimental of greater complexity in ex vivo and in vivo models, it may serve as a clue for hypothesizing therapeutic 
approaches which exploits proteasome hyperactivation increasing the rate of degradation of selected targets through non-conventional drugs such as Proteolysis Targeted Chimeras (PROTACs).

\section{Materials And Methods}

Cells and cell culture conditions

The immortalized rat retinal Müller cells (rMC-1) were obtained from Kerafast (Kerafast, Boston, MA, USA) 57 and were maintained in $5 \mathrm{mmol} / \mathrm{L}$ glucose medium (Low Glucose DMEM), supplemented with $10 \%$ FBS plus antibiotics (Lonza, Basel, Switzerland). The challenge with $25 \mathrm{mmol} / \mathrm{L}$ glucose (referred to as high glucose throughout the text) was performed by delivering either High Glucose DMEM, or Low Glucose DMEM supplemented with D+ Glucose powder $(20 \mathrm{mmol} / \mathrm{L})$. The two treatments provided fully overlapping results. Hyperosmolar stress was induced by delivering Low Glucose DMEM supplemented with $20 \mathrm{mmol} / \mathrm{L}$ mannitol. Remarkably, data from low glucose and mannitol treated cells were fully overlapping, confirming that osmolarity does not affect rMC1 activation, as commonly described by several authors ${ }^{7-9,47}$. Thus, for the sake of readership, in the case of $\mathrm{Wb}$ analysis, data of low glucose and mannitol-treated cells are not discussed separately, unless otherwise indicated, and are generally referred to as untreated/control cells. In the uncropped file, main data including mannitol lanes are shown. In all cases, stimuli were delivered on cells grown at 70\% confluency.

Proteasome Assay, Native Gel Electrophoresis and TED fluorescence in vivo

Cells were lysed under non-denaturing conditions by freeze-thawing cycles in $250 \mathrm{mM}$ sucrose, $20 \%$ glycerol, $25 \mathrm{mM}$ Tris-HCl, 5 mM MgCl $2,1 \mathrm{mM}$ EDTA, 1 mM DTT, 2 mM ATP, pH 7.4, as described elsewhere 58,59 . Thereafter, lysates were cleared by centrifugation at $13,000 \mathrm{rpm}, 20 \mathrm{~min}, 4^{\circ} \mathrm{C}$ and protein concentration determined by Bradford assay.

For proteasome assays, $20 \mu \mathrm{g}$ of proteins were diluted in $20 \%$ glycerol, $25 \mathrm{mM}$ Tris- $\mathrm{HCl}, 5 \mathrm{mM} \mathrm{MgCl} 2,1$ mM EDTA, 1 mM DTT, 2 mM ATP, pH 7.4 in the presence or absence of $500 \mathrm{nM}$ epoxomicin in a Corning 96-well Black Microplate. Reaction mixtures were pre-incubated 30 min at $37^{\circ} \mathrm{C}$. Thereafter, $50 \mu \mathrm{M} 7-$ amino-4-methylcoumarin (AMC) labeled Suc - Leu - Leu - Val - Tyr -AMC peptide (referred to as LLVY-amc) (Boston Biochem, Boston, MA, USA) was delivered to each well and the release of fluorescence monitored over 40 min-1h (in any case, until linearity was observed) in a Cary Eclipse Varian spectrofluorimeter. Values obtained, representative of the moles of substrate processed per time (indicated as nmol substrate/min) were calculated and plotted. The rate of peptide hydrolysis (negligible) in the presence of epoxomicin was subtracted from that in the absence of the proteasome inhibitor. Each experimental condition was run in triplicate into the same plate. The slopes of each curve were then figured out and compared at each time-point.

For native-gel electrophoresis, $75 \mu \mathrm{g}$ of proteins from each experimental condition were separated under native conditions into a 3.5\% polyacrylamide gel. Gels were then soaked in in reaction buffer (50 mM Tris, 
$5 \mathrm{mM} \mathrm{MgCl}_{2}, 1 \mathrm{mM}$ ATP, pH 7.5) supplemented with $75 \mu \mathrm{M}$ LLVY-amc. Complexes were then transferred to a HyBond-ECL nitrocellulose filters (Amersham Biosciences, Amersham, UK) after a mild denaturation in 1\% SDS $25 \mathrm{mM}$ Tris-HCl and probed with an anti-Rpt5, -Rpt3 (Protein-tech Group, Manchester, UK), or -a4 (EnzoLife Science, London, UK) antibodies, diluted 1:3000 in 0.02\% Tween-PBS plus fat-free milk and, thereafter, incubated with a Horseradish Peroxidase-conjugated anti-rabbit or anti-mouse IgG antibody (Biorad, Hercules, CA, USA), diluted 1:50,000 in 0.2\% Tween-PBS fat-free milk.

The release of TED (a generous gift of prof. Mario Salmona, IRCCS Mario Negri, Milan, Italy) fluorescence by living $\mathrm{rMC} 1$ was determined as previously described ${ }^{32}$. Briefly, cells were pretreated with $500 \mathrm{nM}$ epoxomicin or equivalent volume of DMSO for $1 \mathrm{~h}$. The compounds were washed out and high glucose or control media were delivered. After 5 min of incubation $17 \mu \mathrm{M}$ TED was added to the cell culture and fluorescence release recorded in a Tecan Spark spectro-fluorimeter over 20 min from delivery.

Western blotting

For denaturing and reducing western blotting $(\mathrm{Wb})$, cell pellets were processed through different approaches depending on the target.

For unphosphorylated proteins, cell pellets were lysed in RIPA buffer supplemented with cocktails of proteases and phosphatases inhibitors and cleared by centrifugation at $13.000 \mathrm{rpm}$ for $30 \mathrm{~min}$, at $4^{\circ} \mathrm{C}$. Protein concentration was determined by Bradford assay. Depending on the target, 2 up to $40 \mu \mathrm{g}$ of proteins per lane were loaded.

For phosphorylated proteins analysis, cells were directly lysed in 2x Laemmli buffer supplemented with 1 $\mathrm{mM}$ DTT, vortexed $1 \mathrm{~min}$ at maximum speed and heat-denatured $10 \mathrm{~min}$ at $95^{\circ} \mathrm{C}$. This lysis method was preferred also for analysis through phos-Tag polyacrylamide gels performed following manufacturer's instructions (Wako Films, Chemicals, Richmond, VA, USA). Notably, phos-Tag acrylamide refers to an electrophoretic procedure based on a synthetic acrylamide which delays the electrophoretic migration of phosphorylated proteins. Protein separation is followed by canonical Wb procedure which allows to stain the phosphorylated and unphosphorylated species of any given protein.

Different acrylamide concentrations were tested and the best results were obtained by using 15\% polyacrylamide-gels supplemented with $50 \mu \mathrm{M}$ of phos-Tag reagent.

Canonical western blotting was set up depending on the target: a range of $10 \%-15 \%$ polyacrylamide gels were used. A 15\% composition furnished better quality images in the case of pRpt6(ser120). In this case, Ponceau S staining was not performed, as this treatment (probably, Ponceau de-staining with strong bases) was shown to impair protein detection. Conversely, plkBa(ser32) immunostaining was unaffected by Ponceau staining. Whilst blocking procedure was generally performed as described in the previous section, that of filters probed with the anti-Rpt6(ser120) antibody (Mybiosource, San Diego, CA; USA) were processed as it follows: non-specific binding sites were saturated with a $2.5 \%$ albumin (Sigma-Aldrich, StLouis, Co, USA) and 2.5\% fatty-free milk dissolved in Tris-HCl Buffered Saline (TBS) pH 7.5 plus 0.05\% 
Tween for $3 \mathrm{~h}$ at room temperature. The antibody was diluted 1:1000 in the same solution, and incubated overnight at $4^{\circ} \mathrm{C}$. Subsequent procedures were performed as previously indicated. Antibodies used for NF$\mathrm{kB}$ signalling pathway and for CamKII immunodetection were all purchased from Cell Signalling (London, UK). The antibody named pan-a20S was purchased from EnzoLife Science (London, UK). Antibodies raised against cytokines were purchased from Abclonal (Woburn, MA, USA).

As a general note for the Western blotting analysis herein studied, since preliminary settings showed that, in most cases, the micrograms loaded fall off the dynamic linear range of canonical antibodies used as internal controls (e.g., tubulin, actin, GAPDH), even though internal controls lanes are generally reported, normalization was performed on Ponceau S staining unless otherwise indicated (see Suppl. Fig. 5). The choice of actin or GAPDH was dictated by the overlapping molecular weights of these internal controls with the proteins here studied (IkBa and GAPDH show similar MW).

Densitometric analysis of the bands was performed through ImageJ Quant Software. Unless otherwise indicated (see Fig. 7), original figures manipulation was limited to adjustments of light/contrast by using the specific command or the "curve" tool of GIMP2.0 software.

Gene expression analysis

RNA was isolated with Trizol reagent (Life-tecnologies). First strand cDNAs were synthesized from $1 \mu \mathrm{g}$ of total RNA in a $20 \mu \mathrm{l}$ reaction with reverse transcriptase according to manufacturer instructions (BioLine, London, UK). Real-time PCR was performed on $30 \mathrm{ng}$ of cDNA, using a SYBR green Master Mix (Biorad, Hercules, CA, USA). $\beta$-actin was used as internal control. All primers used in these experiments are reported in Table 1.

\section{Rpt6 mutagenesis}

PT-Rex-DEST30 control vector and PT-Rex-DEST30, in which Rat Rpt6 cDNA with a phospho-dead mutation serine to alanine (S120A) was cloned, were purchased from Life Technologies (London, UK). Plasmids were re-suspended in $10 \mu \mathrm{l}$ of sterile water to prepare $1 \mu \mathrm{g} / \mu \mathrm{l}$ stock solution and stored at $-20 \mathrm{C}^{\circ}$ until use. One day before transfection, rMC1 cells were seeded at a density of 4-6 x 104 per well on a 6well plate in complete growth medium to obtain $50-70 \%$ confluence the following day. The day of transfection, $1 \mu \mathrm{g}$ of DNA (PT-Rex-DEST30 control vector and PT-Rex-DEST30-Rpt6(S120A)) was diluted into Turbofectin transfection reagent (OriGene, Rockville, MA, USA) and added to seeded cells according to manufacturer's instructions. Then, cells were incubated for 48 hours and after, cells were splitted (at 1:10 or higher dilution) into fresh growth medium containing selective medium. The selection was performed using $1000 \mu \mathrm{g} / \mathrm{ml}$ geneticin (Sigma Aldrich, St. Louis, MI, USA) for two weeks. Different resistant clones for each plasmid were selected and routinely grown in selective medium containing 300 $\mu \mathrm{g} / \mathrm{ml}$ geneticin.

For induction experiments with tetracycline, transformed cells were seeded at a density of 7-10 $\times 10^{4}$ per well on a 6-well plate in selective medium containing $300 \mu \mathrm{g} / \mathrm{ml}$ geneticin. The following day, 0,5 $\mu \mathrm{g} / \mathrm{ml}$ 
of tetracycline (Sigma Aldrich, St. Louis, MI, USA) was added and incubated for $24 \mathrm{~h}$ at $37 \mathrm{C}^{\circ}$.

\section{Data analysis and statistics}

In all cases, values reported are expressed as mean \pm SD. One-way ANOVA followed by Tukey post-hoc significance test and $\tau$ Student's test were used to calculate statistically significant differences between groups. Unless otherwise indicated, comparisons were made between low-glucose/mannitol treated cells and high glucose treated cells. Data of $\mathrm{Wb}$ immunoreactive bands were presented as relative ratio and a nominal value of 1 was assigned to the first lane of the filter. To simplify the statistical analysis reported, * refers to $p$ values between 0.05 and 0.01 , ** to $p$ values between 0.01 and 0.001 , and *** to $p$ values < 0.001 .

Statistics were calculated by GraphPad software 9.0.

\section{Declarations}

\section{Ethics approval and consent to participate}

This study did not involve human subjects of animal models.

\section{Consent for publication}

Publisher has the author's permission to publish research findings.

\section{Availability of data and material}

Original data will be made available upon reasonable request to M.D. Ph. D. Diego Sbardella, uncropped gel gels are available online as a separate pdf file.

\section{Competing Interest}

The authors declare no conflict of interest

\section{Funding}

The authors acknowledge the financial support by Fondazione Roma and Laziolnnova (Project n. A03752020-36591 to M.C.).

\section{Authors contribution}

D.S., G.R.T. designed the project, performed the experiments and wrote the manuscript, M.P., designed the project, funded it, wrote the manuscript, M.V., M.C., G.G., P.M.L., A.M.C., C.P., P.C., discussed the data, and designed experiments execution, M.G.A., A.M., L.L. performed the experiments. 
Authors are grateful to Prof. Mario Salmona for the generous gift of TED peptide.

\section{References}

1 Wang W, Lo ACY. Diabetic Retinopathy: Pathophysiology and Treatments. Int J Mol Sci 2018; 19. doi:10.3390/ijms19061816.

2 Duh EJ, Sun JK, Stitt AW. Diabetic retinopathy: current understanding, mechanisms, and treatment strategies. JCI Insight 2017; 2. doi:10.1172/jci.insight.93751.

3 Zong H, Ward M, Madden A, Yong PH, Limb GA, Curtis TM et al. Hyperglycaemia-induced proinflammatory responses by retinal Müller glia are regulated by the receptor for advanced glycation endproducts (RAGE). Diabetologia 2010; 53: 2656-2666.

4 Coughlin L, Morrison RS, Horner PJ, Inman DM. Mitochondrial morphology differences and mitophagy deficit in murine glaucomatous optic nerve. Invest Ophthalmol Vis Sci 2015; 56: 1437-1446.

5 Mizutani M, Gerhardinger C, Lorenzi M. Müller cell changes in human diabetic retinopathy. Diabetes 1998; 47: 445-449.

6 Lupien CB, Bolduc C, Landreville S, Salesse C. Comparison between the gene expression profile of human Müller cells and two spontaneous Müller cell lines. Invest Ophthalmol Vis Sci 2007; 48: 52295242.

7 Picconi F, Parravano M, Sciarretta F, Fulci C, Nali M, Frontoni S et al. Activation of retinal Müller cells in response to glucose variability. Endocrine 2019; 65: 542-549.

8 Chang M-L, Chiu C-J, Shang F, Taylor A. High glucose activates ChREBP-mediated HIF-1a and VEGF expression in human RPE cells under normoxia. Adv Exp Med Bio/ 2014; 801: 609-621.

9 Li J, Zhao S, Wang P, Yu S, Zheng Z, Xu X. Calcium mediates high glucose-induced HIF-1a and VEGF expression in cultured rat retinal Müller cells through CaMKII-CREB pathway. Acta Pharmacol Sin 2012; 33: $1030-1036$.

10 Lin M, Chen Y, Jin J, Hu Y, Zhou KK, Zhu M et al. Ischaemia-induced retinal neovascularisation and diabetic retinopathy in mice with conditional knockout of hypoxia-inducible factor-1 in retinal Müller cells. Diabetologia 2011; 54: 1554-1566.

11 Mathes E, O'Dea EL, Hoffmann A, Ghosh G. NF-kB dictates the degradation pathway of IKBa. EMBO J 2008; 27: 1357-1367.

12 Ferreiro DU, Komives EA. Molecular mechanisms of system control of NF-kappaB signaling by IkappaBalpha. Biochemistry 2010; 49: 1560-1567. 
13 Christian F, Smith EL, Carmody RJ. The Regulation of NF-kB Subunits by Phosphorylation. Cells 2016; 5. doi:10.3390/cells5010012.

14 Taniguchi K, Karin M. NF-KB, inflammation, immunity and cancer: coming of age. Nat Rev Immunol 2018; 18: 309-324.

15 Liu T, Zhang L, Joo D, Sun S-C. NF-kB signaling in inflammation. Sig Transduct Target Ther 2017; 2 : 17023.

16 Sun S-C. The non-canonical NF-KB pathway in immunity and inflammation. Nat Rev Immuno/ 2017; 17: $545-558$.

17 Glickman $\mathrm{MH}$, Ciechanover A. The ubiquitin-proteasome proteolytic pathway: destruction for the sake of construction. Physiol Rev 2002; 82: 373-428.

18 Livneh I, Cohen-Kaplan V, Cohen-Rosenzweig C, Avni N, Ciechanover A. The life cycle of the 26S proteasome: from birth, through regulation and function, and onto its death. Cell Res 2016; 26: 869-885.

19 Tundo GR, Sbardella D, Santoro AM, Coletta A, Oddone F, Grasso G et al. The proteasome as a druggable target with multiple therapeutic potentialities: Cutting and non-cutting edges. Pharmacol Ther 2020; 213: 107579.

20 Collins GA, Goldberg AL. The Logic of the 26S Proteasome. Cel/ 2017; 169: 792-806.

21 Davies KJ. Degradation of oxidized proteins by the 20 S proteasome. Biochimie 2001; 83: 301-310.

22 Lander GC, Estrin E, Matyskiela ME, Bashore C, Nogales E, Martin A. Complete subunit architecture of the proteasome regulatory particle. Nature 2012; 482: 186-191.

23 Martin A, Baker TA, Sauer RT. Pore loops of the AAA+ ClpX machine grip substrates to drive translocation and unfolding. Nat Struct Mol Biol 2008; 15: 1147-1151.

24 Bard JAM, Goodall EA, Greene ER, Jonsson E, Dong KC, Martin A. Structure and Function of the $26 \mathrm{~S}$ Proteasome. Annu Rev Biochem 2018; 87: 697-724.

25 Kincaid EZ, Che JW, York I, Escobar H, Reyes-Vargas E, Delgado JC et al. Mice completely lacking immunoproteasomes show major changes in antigen presentation. Nat Immuno/ 2011; 13: 129-135.

26 Groettrup M, Kirk CJ, Basler M. Proteasomes in immune cells: more than peptide producers? Nat Rev Immunol 2010; 10: 73-78.

27 Raule M, Cerruti F, Cascio P. Enhanced rate of degradation of basic proteins by $26 \mathrm{~S}$ immunoproteasomes. Biochim Biophys Acta 2014; 1843: 1942-1947. 
28 Cascio P, Call M, Petre BM, Walz T, Goldberg AL. Properties of the hybrid form of the 26S proteasome containing both $19 \mathrm{~S}$ and PA28 complexes. EMBO J 2002; 21: 2636-2645.

29 Cascio P. PA28aß: the enigmatic magic ring of the proteasome? Biomolecules 2014; 4: 566-584.

30 VerPlank JJS, Goldberg AL. Regulating protein breakdown through proteasome phosphorylation. Biochem J 2017; 474: 3355-3371.

31 VerPlank JJS, Goldberg AL. Exploring the regulation of proteasome function by subunit phosphorylation. Methods Mol Biol 2018; 1844: 309-319.

32 Urru SAM, Veglianese P, De Luigi A, Fumagalli E, Erba E, Gonella Diaza R et al. A new fluorogenic peptide determines proteasome activity in single cells. J Med Chem 2010; 53: 7452-7460.

33 VerPlank JJS, Lokireddy S, Zhao J, Goldberg AL. 26S Proteasomes are rapidly activated by diverse hormones and physiological states that raise cAMP and cause Rpn6 phosphorylation. PNAS 2019; 116: 4228-4237.

34 Djakovic SN, Schwarz LA, Barylko B, DeMartino GN, Patrick GN. Regulation of the proteasome by neuronal activity and calcium/calmodulin-dependent protein kinase II. J Biol Chem 2009; 284: 2665526665.

35 Djakovic SN, Marquez-Lona EM, Jakawich SK, Wright R, Chu C, Sutton MA et al. Phosphorylation of Rpt6 regulates synaptic strength in hippocampal neurons. J Neurosci 2012; 32: 5126-5131.

36 Jarome TJ, Kwapis JL, Ruenzel WL, Helmstetter FJ. CaMKII, but not protein kinase A, regulates Rpt6 phosphorylation and proteasome activity during the formation of long-term memories. Front Behav Neurosci 2013; 7. doi:10.3389/fnbeh.2013.00115.

37 Lokireddy S, Kukushkin NV, Goldberg AL. cAMP-induced phosphorylation of 26S proteasomes on Rpn6/PSMD11 enhances their activity and the degradation of misfolded proteins. Proc Natl Acad Sci US A 2015; 112: E7176-E7185.

38 Sbardella D, Tundo GR, Coletta A, Marcoux J, Koufogeorgou El, Ciaccio C et al. The insulin-degrading enzyme is an allosteric modulator of the $20 \mathrm{~S}$ proteasome and a potential competitor of the 19S. Cell $\mathrm{Mol}$ Life Sci 2018; 75: 3441-3456.

39 Sbardella D, Tundo GR, Sciandra F, Bozzi M, Gioia M, Ciaccio C et al. Proteasome Activity Is Affected by Fluctuations in Insulin-Degrading Enzyme Distribution. PLOS ONE 2015; 10: e0132455.

40 Simó R, Stitt AW, Gardner TW. Neurodegeneration in diabetic retinopathy: does it really matter? Diabetologia 2018; 61: 1902-1912. 
41 Metea MR, Newman EA. Glial cells dilate and constrict blood vessels: a mechanism of neurovascular coupling. J Neurosci 2006; 26: 2862-2870.

42 Newman EA. Glial cell regulation of neuronal activity and blood flow in the retina by release of gliotransmitters. Philos Trans R Soc Lond B Biol Sci 2015; 370. doi:10.1098/rstb.2014.0195.

43 Shi H, Berger EA. Characterization of Site-Specific Phosphorylation of NF-kB p65 in Retinal Cells in Response to High Glucose and Cytokine Polarization. Mediators Inflamm 2018; 2018. doi:10.1155/2018/3020675.

44 Le Y-Z. VEGF production and signaling in Müller glia are critical to modulating vascular function and neuronal integrity in diabetic retinopathy and hypoxic retinal vascular diseases. Vision Res 2017; 139: 108-114.

45 Li W, Liu X, Tu Y, Ding D, Yi Q, Sun X et al. Dysfunctional Nurr1 promotes high glucose-induced Müller cell activation by up-regulating the NF-KB/NLRP3 inflammasome axis. Neuropeptides 2020; 82: 102057.

46 Matteucci A, Gaddini L, Villa M, Varano M, Parravano M, Monteleone V et al. Neuroprotection by rat Müller glia against high glucose-induced neurodegeneration through a mechanism involving ERK1/2 activation. Exp Eye Res 2014; 125: 20-29.

47 Trueblood KE, Mohr S, Dubyak GR. Purinergic regulation of high-glucose-induced caspase-1 activation in the rat retinal Müller cell line rMC-1. Am J Physiol Cell Physiol 2011; 301: C1213-1223.

48 Asano S, Fukuda Y, Beck F, Aufderheide A, Förster F, Danev R et al. Proteasomes. A molecular census of 26 S proteasomes in intact neurons. Science 2015; 347: 439-442.

49 Bianchi G, Oliva L, Cascio P, Pengo N, Fontana F, Cerruti F et al. The proteasome load versus capacity balance determines apoptotic sensitivity of multiple myeloma cells to proteasome inhibition. Blood 2009; 113: $3040-3049$.

50 Alverdi V, Hetrick B, Joseph S, Komives EA. Direct observation of a transient ternary complex during IKBa-mediated dissociation of NF-kB from DNA. Proc Natl Acad Sci U S A 2014; 111: 225-230.

51 Çetin G, Klafack S, Studencka-Turski M, Krüger E, Ebstein F. The Ubiquitin-Proteasome System in Immune Cells. Biomolecules 2021; 11: 60.

52 Maldonado M, Kapphahn RJ, Terluk MR, Heuss ND, Yuan C, Gregerson DS et al. Immunoproteasome deficiency modifies the alternative pathway of NFKB signaling. PLoS One 2013; 8: e56187.

53 Marquez-Lona EM, Torres-Machorro AL, Gonzales FR, Pillus L, Patrick GN. Phosphorylation of the 19S regulatory particle ATPase subunit, Rpt6, modifies susceptibility to proteotoxic stress and protein aggregation. PLoS One 2017; 12: e0179893. 
54 Ben-Nissan G, Sharon M. Regulating the 20S Proteasome Ubiquitin-Independent Degradation Pathway. Biomolecules 2014; 4: 862-884.

55 Scudder SL, Gonzales FR, Howell KK, Stein IS, Dozier LE, Anagnostaras SG et al. Altered Phosphorylation of the Proteasome Subunit Rpt6 Has Minimal Impact on Synaptic Plasticity and Learning. eNeuro 2021; 8: ENEURO.0073-20.2021.

56 Cenci S, Oliva L, Cerruti F, Milan E, Bianchi G, Raule M et al. Pivotal Advance: Protein synthesis modulates responsiveness of differentiating and malignant plasma cells to proteasome inhibitors. $J$ Leukoc Biol 2012; 92: 921-931.

57 Sarthy VP, Brodjian SJ, Dutt K, Kennedy BN, French RP, Crabb JW. Establishment and characterization of a retinal Müller cell line. Invest Ophthalmol Vis Sci 1998; 39: 212-216.

58 Elsasser S, Schmidt M, Finley D. Characterization of the proteasome using native gel electrophoresis. Meth Enzymo/ 2005; 398: 353-363.

59 Sbardella D, Coletta A, Tundo GR, Ahmed IMM, Bellia F, Oddone F et al. Structural and functional evidence for citicoline binding and modulation of 20S proteasome activity: Novel insights into its proproteostatic effect. Biochem Pharmacol 2020; 177: 113977.

\section{Tables}

Table 1. List of primers used for RT-PCR analysis.

\begin{tabular}{|c|c|}
\hline Gene & Sequence \\
\hline \multirow[t]{2}{*}{$\beta$-actin } & 5'- AGCCATGTACGTAGCCATCC-3' \\
\hline & 5'-CTCTCAGCTGTGGTGGTGAA-3' \\
\hline \multirow[t]{2}{*}{$I L-1 \beta$} & 5'ACTCATTGTGGCTGTGGAGA-3 \\
\hline & 5'-TAGCAGGTCGTCATCATCCC-3' \\
\hline \multirow[t]{2}{*}{ IL-6 } & 5'-TGATGGATGCTTCCAAACTG-3' \\
\hline & 5'-GAGCATTGGAAGTTGGGGTA-3' \\
\hline \multirow[t]{2}{*}{ IL-8 } & 5'-GAAGATAGATTGCACCGA-3' \\
\hline & 5'-CATAGCCTCTCACACATTTC-3' \\
\hline \multirow[t]{2}{*}{ MCP-1 } & 5'- GCCAGTGAATGAGTAGCAAG-3' \\
\hline & 5'-CTTCTGGGCCTGTTGTTCAC-3' \\
\hline \multirow[t]{2}{*}{$T N F-a$} & 5-'ACTGAACTTCGGGGTGATTG-3' \\
\hline & 5-'GCTTGGTGGTTTGCTACGAC-5' \\
\hline
\end{tabular}

Page 21/26 


\section{Figures}

\section{Figure 1}

The transcription of proinflammatory cytokines in $\mathrm{rMC1}$ cells is quickly induced by high glucose. (A) Whole cell extracts of $\mathrm{rMC} 1$ cells challenged with $25 \mathrm{mmol} / \mathrm{L}$ glucose and $5 \mathrm{mmol} / \mathrm{L}$ glucose or 20 $\mathrm{mmol} / \mathrm{L}$ mannitol (a representative low glucose lane is reported in all figures showing immunoblots) for the indicated time-points were analysed by $\mathrm{Wb}$ (labelled as "IB" throughout all figures) and probed with antibodies raised against IL-1 $\beta$ and IL-12. Total proteins stained by Ponceau S were used as loading control. Histograms show the densitometric analysis of the immunoreactive bands; data are the mean $\pm S D$ of three independent experiments; a representative blot is shown. Statistics refer to the comparison between high and low glucose treated cells. One-way ANOVA followed by Tukey's post-hoc test: $\star \star \star p<0.001$; (B) RT-PCR analysis of a selection of proinflammatory cytokines in the same sample groups as in (A). Transcript levels of high glucose treated cells were compared to low glucose and mannitol treated cells. $\beta$-actin was used as internal control. Data are presented as mean $\pm S D(n=3)$; one-way ANOVA followed by Tukey's post-hoc test $(n=3)$ : ${ }^{* \star} p<0.01$, ${ }^{\star \star \star} p<0.001$.

\section{Figure 2}

High glucose stimulation induces the nuclear translocation of p65-p50 regardless of $1 \mathrm{kBa}$ phosphorylation. (A) Whole cell lysates were prepared from rMC1 cells challenged with $25 \mathrm{mmol} / \mathrm{L}$ glucose for 10, 20 and 40 min or from control cells and analysed by Wb. Filters were probed with antibodies anti-plkBa(ser32) and - $\mathrm{kBa}$. Total proteins stained by Ponceau $\mathrm{S}$ were used as loading control. Results of densitometric analysis are presented as Mean $\pm S D(n=3)$; a representative blot is shown. Statistics were calculated for high glucose vs low glucose treated cells. One-way ANOVA followed by Tukey's post-hoc test: ${ }^{*} p<0.01,{ }^{*} *{ }^{*} p<0.001$; (B) Phos-tag analysis of the same samples as in (A). The filter was probed with an anti-lkBa antibody. Low and high exposures of the filter are shown; (C) Nuclear and cytosolic extracts were isolated from $\mathrm{rMC} 1$ cells challenged with $25 \mathrm{mmol} / \mathrm{L}$ glucose and low glucose or mannitol for 30 min (preliminary settings showed that stimulation for 30 min provided results more robust than those observed after $20 \mathrm{~min}$ ). Wb analyses of these fractions was performed by using antibodies anti-p65, -p50, -lamin-B2 and $\beta$-tubulin. Histograms represent the results of densitometric analysis of the immunoreactive bands. The intensity of p65 and p50 bands of high glucose treated cells was compared to that of low glucose treated cells separately for each compartment (e.g., nucleus or cytosol); (D) Nuclear extracts from rMC1 cells challenged with $5 \mathrm{mmol} / \mathrm{L}$ glucose, $25 \mathrm{mmol} / \mathrm{L}$ glucose or mannitol were analysed by Trans-AM immune-enzymatic assay and DNA binding by p65 was evaluated as O.D. read at $470 \mathrm{~nm}$. Statistics were calculated for high glucose vs low glucose or mannitol treated cells in (C) and (D) and data are presented as Mean $\pm S D(n=3)$; one-way ANOVA followed by Tukey's posthoc test: ${ }^{*} p<0.05,{ }^{* \star} p<0.01,{ }^{* \star *} p<0.001$. 


\section{Figure 3}

A second cycle of NF-kB activation by high glucose occurs through a typical signalling pathway. Whole cell lysates were prepared from $\mathrm{rMC} 1$ cells challenged with $25 \mathrm{mmol} / \mathrm{L}$ glucose and with low glucose or mannitol for 1, 2 and $3 \mathrm{~h}$ and analysed by Wb. Filters were probed with antibodies anti-plkBa(ser32), - $1 \mathrm{kBa}$, -pIKKß(ser80), -IKKß, -p65(ser536), and -p65. The plkBa(ser32)/lkBa and p65(ser536)/p65 ratios are shown. Being pIKK $\beta$ undetectable in control cells, the unphosphorylated/phosphorylated ratio of this protein is not reported. Total proteins stained by Ponceau $\mathrm{S}$ were used as loading control. Histograms represent the results of densitometric analysis of the immunoreactive bands; data are the mean \pm SD of three independent experiments; a representative blot is shown. Statistics were calculated for high glucose vs low glucose treated cells using a One-way ANOVA followed by Tukey's post-hoc test: * $p<0.05$; $\star \star \star p<0.001$.

\section{Figure 4}

Proteasome bulk activity is quickly stimulated by high glucose delivery. (A) Whole cell extracts of rMC1 cells stimulated with $25 \mathrm{mmol} / \mathrm{L}$ glucose and with low glucose or mannitol for 10,20 and 40 min were probed with antibodies anti-ubiquitin, -p21 and p53; GAPDH was used as loading control by Wb analysis. Histograms show the densitometric analysis of the immunoreactive bands; data are the mean $\pm S D$ of three independent experiments; a representative blot is shown. Statistics were calculated for high glucose vs low glucose treated cells; one-way ANOVA followed by Tukey's post-hoc test: ${ }^{*} p<0.05,{ }^{\star \star} p<0.01$; (B) rMC1 cells were pre-treated with $500 \mathrm{nM}$ epoxomicin or vehicle (DMSO) for $1 \mathrm{~h}$. Thereafter, the compound was washed out before addition of high glucose or controls. After 40 min of incubation, whole cell lysates were prepared, analysed by $\mathrm{Wb}$ and probed with the anti-ubiquitin antibody. GAPDH was used as loading control; raw normalized values of band intensity are reported below the upper panel; (C) Crude cell extracts were isolated from rMC1 cells challenged with high glucose as indicated in (A) and bulk proteasome activity assayed on Suc-LLVY-amc, a fluorogenic peptide specific for the chymotrypsin-like activity. Values reported in the graph are the relative ratio of the slope calculated for high-glucose treated cells vs low-glucose or mannitol-treated cells at each time-point. Peptide cleavage was monitored until linearity was observed ( $40 \mathrm{~min})$. The time $(\mathrm{x})$ scale refers to the timing after glucose stimulation; slopes have been determined by subtracting that obtained in the presence of $500 \mathrm{nM}$ epoxomicin (added to the crude cell extract) for each experimental condition. Non-specific cleavage of the fluorogenic probe in the presence of epoxomicin was negligible. One-way ANOVA followed by Tukey's post-hoc test $(n=3)$ : ${ }^{*}<<0.05$; $\star \star p<0.01$; (D) Crude cell extracts of cells treated with low glucose and high glucose were separated by native-gel electrophoresis. The activity of proteasome particles was visualized in-gel upon delivery of 75 $\mu \mathrm{M}$ Suc-LLVY-amc. The identity of capped species was determined by $\mathrm{Wb}$ analysis, probing the filters with an anti-Rpt5 antibody. An aliquot of these extracts was further analysed by denaturing and reducing $\mathrm{Wb}$ and probed with anti-a4, -Rpt5 and -Rpt6 antibodies; a representative experiment of three independent 
replicates is reported; (E) living rMC1 cells were pre-stimulated with $500 \mathrm{nM}$ epoxomicin or DMSO for $1 \mathrm{~h}$. Thereafter, the compounds were washed out and the stimuli (i.e., low glucose, high glucose and mannitol) delivered. After additional 5 min of incubation the TED fluorogenic peptide $(17 \mu \mathrm{M})$ was added to the culture medium and fluorescence release monitored over $20 \mathrm{~min}$ of high glucose stimulation. Arrows indicate the precise timing of stimuli administration. Data are reported as relative comparison between Relative Fluorescence Unit (RFU) obtained at each time-point. In accordance with previous results, preliminary data indicated that TED half-life is quite short (around $30 \mathrm{~min}$. and that non-specific cleavage of the peptide was almost null, since epoxomicin treated cells did not release appreciable fluorescence (data not shown) 32. One-way ANOVA followed by Tukey's post-hoc test $(n=3):{ }^{*} p<0.01$.

\section{Figure 5}

Proteasome activation is triggered by phosphorylation of Rpt6 at serine 120 . Whole cell extracts were obtained from rMC1 challenged with $25 \mathrm{mmol} / \mathrm{L}$ glucose and low glucose or mannitol for 20 and $40 \mathrm{~min}$ and analysed by phos-Tag assay and Wb. Phos-Tag filters were probed with antibodies anti-Rpn6, -Rpt5, Rpt3 and -Rpt6. Rpn6 and Rpt5 blots are purposely shown overexposed to highlight the lack of species with delayed electrophoretic mobility; notably, Rpt5 migrated as a doublet band also in denaturing and reducing conditions (see Fig. 4). (B) Wb filters were probed with a phospho-specific antibody raised against Rpt6 phosphorylated at serine 120 [pRpt6(ser120] and with an anti-Rpt6 antibody. The pRpt6(ser120)/Rpt6 ratio between immunoreactive bands was then calculated and reported in histograms. Total proteins stained by Ponceau $S$ were used as loading control. Data are presented as mean $\pm S D(n=3)$; a representative blot is shown. One-way ANOVA followed by Tukey's post-hoc test: $\star \star \star p<0.001$.

\section{Figure 6}

CamKII, but not PKA, phosphorylates Rpt6. (A) rMC1 cells were pre-treated with $10 \mu \mathrm{M}$ DMSO, Inh. XII or KT2750 for $2 \mathrm{~h}$. Thereafter, compounds were washed out and $25 \mathrm{mmol} / \mathrm{L}$ glucose or low glucose/mannitol was added to culture media for $20 \mathrm{~min}$ and crude cell extracts were prepared. Cells treated with low glucose and mannitol were used as controls. Bulk chymotrypsin like activity was determined as described in Fig. 4C and results expressed as relative activity respect to the slope of LLVYamc cleavage by proteasome in crude cell extracts of low glucose stimulated cells. One-way ANOVA followed by Tukey's post-hoc test $(n=3)$ : ${ }^{*} p<0.01$; (B) The same crude cell extracts were also run by native-gel electrophoresis (as in Fig. 4D) and the filters probed with the anti-Rpt5 antibody. An aliquot of the sample was run by denaturing and reducing $\mathrm{Wb}$ to rule out uneven gel loading (bottom panel). (C-D) Whole cell extracts were obtained from rMC1 challenged with $25 \mathrm{mmol} / \mathrm{L}$ for 20 and analysed by phosTag assay (C) and $\mathrm{Wb}(\mathrm{D})$. Phos-Tag filters were probed with the anti-Rpt6 antibody. Wb filters were probed with the phospho-specific anti-Rpt6(ser120) antibody and with the anti-Rpt6 antibody. The pRpt6(ser120)/Rpt6 ratio was then calculated from immunoreactive bands of high glucose vs low 
glucose lanes and shown in histograms. Total proteins stained by Ponceau $\mathrm{S}$ were used as loading control. Data are presented as mean $\pm S D(n=3)$, a representative blot of three independent replicates is shown. One-way ANOVA followed by Tukey's post-hoc test: ${ }^{\star \star *} p<0.001$.

\section{Figure 7}

Treatment with CamKII and proteasome inhibitors prevents NF-kB from being activated by high glucose. (A) rMC1 cells were pre-treated with $10 \mu \mathrm{M}$ DMSO, Inh. XII, KT570 or BAPTA-AM for $2 \mathrm{~h}$ and with $500 \mathrm{nM}$ epoxomicin for $1 \mathrm{~h}$. Thereafter, compounds were washed out and $25 \mathrm{mmol} / \mathrm{L}$ glucose and low glucose/mannitol were added to culture media. After $20 \mathrm{~min}$, whole cell lysates were prepared and analysed by Wb. Filters were probed with the anti-IkBa antibody. Total proteins stained by Ponceau $S$ were used as loading control. Band intensity is reported in histograms. Data are presented as mean $\pm S D$ $(n=3)$; a representative blot is shown. Statistics was calculated by comparing each pair low-high glucose for any given compound assayed Multiple $\tau$ Student's tests $* \star \star p<0.001$; The bands shown belong to the same gel, but this figure has been manipulated to clear out two lanes which were unnecessary for discussion (see uncropped files); (B) rMC1 cells were pre-treated with $10 \mu \mathrm{M} \mathrm{DMSO}$ and Inh. XII for $2 \mathrm{~h}$ and $500 \mathrm{nM}$ epoxomicin for $1 \mathrm{~h}$. Thereafter, compounds were washed out and $25 \mathrm{mmol} / \mathrm{L}$ glucose and low glucose or mannitol (controls) were added. After $40 \mathrm{~min}$ of stimulation nucleic acids were isolated and the expression of proinflammatory cytokines assayed by RT-PCR. $\beta$-actin was used as internal control. Histogram represents the relative quantity of transcripts calculated for any given cytokine for each experimental group (i.e., low glucose, high glucose and mannitol). Statistics was calculated for each cytokine separately. Data are presented as mean $\pm S D(n=3)$. One-way ANOVA followed by Tukey's posthoc test $(n=3):{ }^{*}<0.05 ; * \star * p<0.001$.

\section{Figure 8}

Inh. XII, but not KT5720, prevent canonical signalling of NF-kB from being activated after $2 \mathrm{~h}$ of high glucose challenge. rMC1 cells were pre-treated with $10 \mu \mathrm{M} \mathrm{KT5720,} \mathrm{Inh.} \mathrm{XII} \mathrm{or} \mathrm{DMSO} \mathrm{for} 2 \mathrm{~h}$. Thereafter, 25 $\mathrm{mmol} / \mathrm{L}$ glucose or the same volume of $5 \mathrm{mmol} / \mathrm{L}$ glucose was administered. Whole cell lysates were prepared at 2 and $3 \mathrm{~h}$ after glucose delivery and analysed by Wb. Filters were probed with the anti$\mathrm{IkBa}($ ser32) antibody. Remarkably, epoxomicin was not enrolled due to increase of plkBa(ser32) at these time-points as indicated in Suppl. Fig. 4. Total proteins stained by Ponceau S were used as loading control. A representative experiment of three independent replicates is shown. Histogram represents the relative levels of $\mathrm{pIBa}(\mathrm{ser} 32)$ protein in the different experimental conditions. One-way ANOVA followed by Tukey's post-hoc student test: ${ }^{\star *} p<0.01,{ }^{* \star \star}<p .0 .001$.

\section{Supplementary Files}

This is a list of supplementary files associated with this preprint. Click to download. 
- Suppl.Material.docx

Page 26/26 